\title{
IC 1613 OB Associations and Star Cluster Candidates
}

J. Borissova

Institute of Astronomy, Bulgarian Academy of Sciences, Sofia, Bulgaria

R. Kurtev

Department of Astronomy, Sofia University, Sofia, Bulgaria

L. Georgiev

Instituto de Astronoma, Universidad Nacional Autonoma de Mexico, Mexico

M. Rosado

Instituto de Astronoma, Universidad Nacional Autonoma de Mexico, Mexico

G. Ivanov

Department of Astronomy, Sofia University, Sofia, Bulgaria

Abstract. Moderately deep ground based $U B V$ images are used to investigate the $\mathrm{OB}$ associations and star clusters in IC 1613. Fifty eight OB associations with sizes between 30 and $130 \mathrm{pc}$ are outlined. The iteraction between associations and superbubbles in IC 1613 was analyzed. The lack of star clusters was confirmed.

\section{Introduction}

The OB associations and clusters in IC 1613 are investigated first by Hodge (1978). He identified twenty OB associations and 43 possible cluster candidates. Freedman (1988) presented color-magnitude diagrams for 11 of Hodge associations. The properties of nine of them located in the NE sector of the galaxy are analyzed by Georgiev et al. (1999) as well as seven new OB associations are determined by cluster analysis. Wyder et al. (2000) surveyed the central area of the IC 1613 for star clusters. They found that there are no globular clusters and six very sparse groups can be open clusters.

The purpose of the present paper is to outline the new boundaries of the associations in IC 1613 using the observational material of uniform quality and objective methods for identification of stellar associations and clusters. 


\section{Approach to the Problem}

A set of $U B V$ frames of IC 1613 were obtained on the 2-m telescopes of the Bulgarian National Astronomical Observatory and Mexican National Astronomical Observatory at San Pedro Martir. The stellar photometry was performed with the point-spread function fitting routine ALLSTAR available in DAOPHOT. The automatized search for $\mathrm{OB}$ associations was carried out using Battinelli (1991) so called friend-of-friends algorithm. The search of resolved star clusters was made by method described in Georgiev et al. (1999). The search of unresolved star clusters was made by routine INVENTORY available in MIDAS.

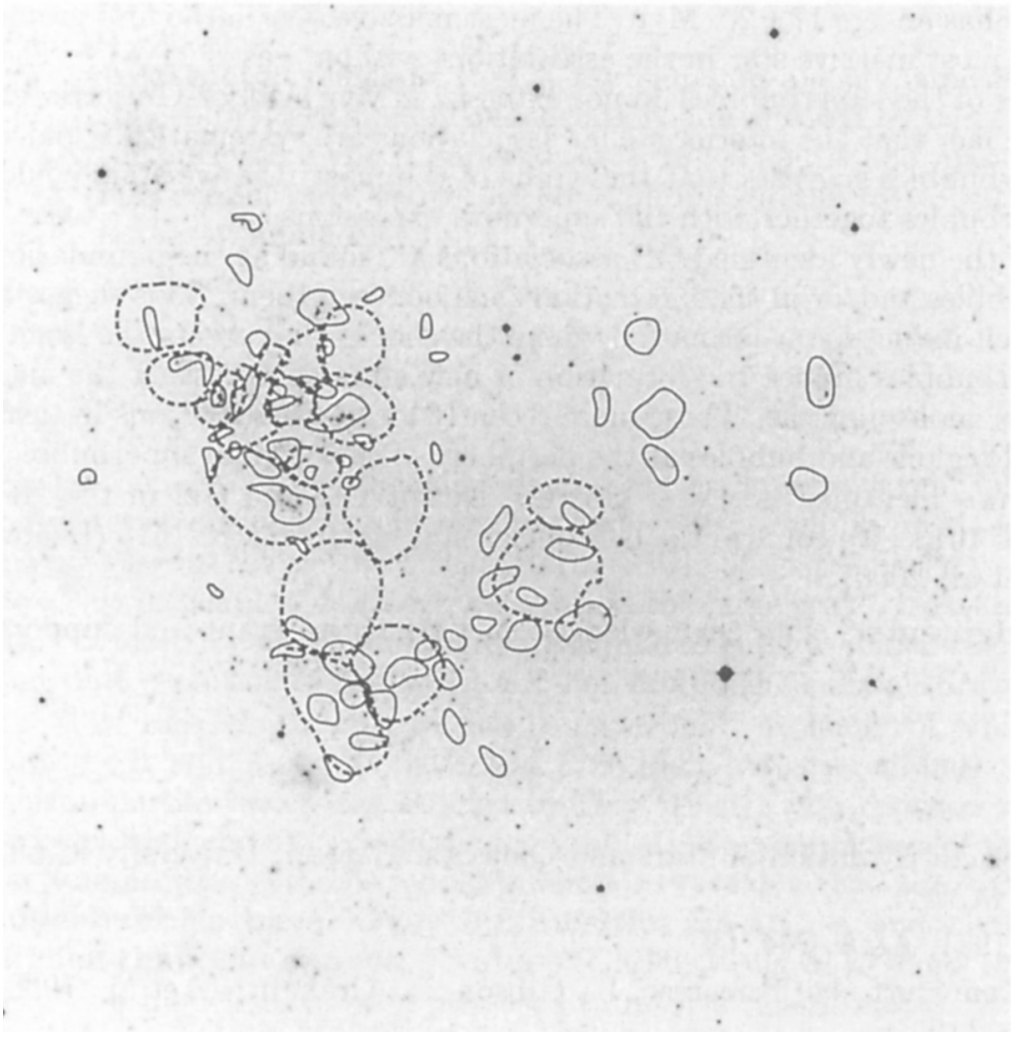

Figure 1. The OB-associations (solid lines) and the superbubbles (dashed lines) in IC 1613

\section{Results}

The Battinelli (1991) algorithm selects 58 associations (Figure 1) with sizes between 30 and $130 \mathrm{pc}$ (accepted distance is $730 \mathrm{kpc}$ (Dolphin et al. 2000, Borissova et al 2000). The mean size of the associations is $64 \pm 11 \mathrm{pc}$, comparable with the mean sizes of associations in the LMC - 60pc, SMC - 70 pc and M33 - 60 pc 
(Bresolin et al. 1998). Most of the stellar associations outlined by Hodge (1978) coincide with our groups, but in general each Hodge association divides into several smaller groups. The associations are not uniformly distributed within the galaxy and are mostly concentrated in the NE and NW regions (Hodge 1978).

The ionized gas in IC 1613 is distributed in large diameter, expanding superbubbles (Valdez-Gutierrez et al. 2000). Almost every superbubble in IC 1613 (dashed lines in Fig. 1) has an interior association (solid lines in Fig. 1). These associations are located within or at the peripheries of the superbubbles and probably they are physically connected with the gas.

There is an age and mass difference between associations connected with the superbubbles and the remaining ones. The age of the first group is $7 \pm 2.5 \mathrm{Myr}$, the second one has an age $17 \pm 2.5 \mathrm{Myr}$. The most massive star in the first group is $60 \mathcal{M}_{\odot}$, the most massive star in the associations without gas is $25 \mathcal{M}_{\odot}$. The dynamical ages of the superbubbles do not extend 1-2 Myr (Valdez-Gutierrez et al. 2000). The fact that the interior stellar associations are systematically older than the superbubbles suggests that the winds of the most massive stars could form the superbubles together with the supernova explosions.

Several of the newly identified OB associations are found at the boundaries of the superbubbles and/or in the interaction zone between them. This suggests a scenario of self-induced star formation where the shocks that create the larger diameter superbubbles induce the formation of new stars compressing the ambient neutral or molecular gas. These more recently formed associations in turn create new HII regions and bubbles at the periphery of the original superbubble.

We have not identified new star clusters, except Cl1 and Cl2 in the NE region of the IC 1613 . We confirm the lack of rich star clusters in IC 1613 (Baade 1963, Wyder et al. 2000).

Acknowledgments. J.B. acknowledges an IAU travel grant and support from the LOC.

\section{References}

Baade, W.,1963, in Evolution of Stars and Galaxies. Harvard University, Cambridge, MA.

Battinelli, P., 1991, A\&A, 244, 69

Bresolin, F., Kennicutt, Jr., Ferrareze, L., Gibson, B., Graham, J., et al., 1998, AJ, 116, 119

Borissova, J., Georgiev, L., Kurtev, R., Rosado, M. et al., 2000, RMxAA, 36, 151

Dolphin, A., Saha, A., Skillman, E., Tolstoy, E., et al., 2000, ApJ, 550, 554

Freedman, W., 1988, AJ, 96, 1248

Georgiev, L., Borissova, J., Rosado, M., Kurtev, R., Ivanov, G., et al., 1999, A\&AS, 134, 21

Hodge,P., 1978, ApJS, 37, 145

Valdez-Gutierrez, M., Rosado, M., Georgiev, L., Borissova, J., Kurtev, R., 2001, $\mathrm{A} \& \mathrm{~A}, 366,35$

Wyder, T., Hodge, P., Cole, A., 2000, PASP, 112, 594 